\title{
Near Resonant Scattering from Nonsymmetric Dimers: Applications to Substituted Polyanilines
}

\author{
F. C. Lavarda, ${ }^{1,2}$ M. C. dos Santos, ${ }^{1}$ D. S. Galvão, ${ }^{1}$ and B. Laks, ${ }^{1}$ \\ ${ }^{1}$ Instituto de Física "Gleb Wataghin," Universidade Estadual de Campinas, 13081-970 Campinas, Sao Paulo, Brazil \\ ${ }^{2}$ Departamento de Física, UNESP-Bauru, 17033 Bauru, Sao Paulo, Brazil
}

(Received 18 January 1994)

\begin{abstract}
In this work we show that, beyond the prediction of the random dimer model [Wu and Phillips, Phys. Rev. Lett. 66, 1366 (1991)], it is possible to have near resonant scattering from nonsymmetric dimers. It is shown by direct density of states calculations as well as by a procedure similar to the random dimer model that protonated chains of alkyl-substituted polyanilines support extended electronic states at the Fermi energy when a disordered distribution of symmetric or asymmetric bipolarons is present. An extension of the random dimer model to include resonant scattering by nonsymmetric dimers is proposed.
\end{abstract}

PACS numbers: 71.20.Hk, 71.30. $+\mathrm{h}, 71.55 . \mathrm{Jv}$

Chemical doping of organic conjugated polymers has been shown to produce drastic modifications in transport and optical properties of these materials, which in some cases undergo an insulator-to-metal transition. This chemical process can be either a charge transfer reaction of strong oxidizing or reducing agents with the polymer chains or an acidic treatment in which protons attach to certain sites of the chains. It is currently accepted that local distortions of the chemical bonds occur in the chains in order to store the extra charge. These distortions are commonly referred to as solitons, polarons, and bipolarons, and the role played by these defects in the conduction mechanism is still an open and polemical question [14]. At high dopant concentrations, regular distributions of these defects have been proposed to explain the transition to a metallic state. However, these models fail in some aspect: A regular distribution of solitons or bipolarons leads to a semiconductorlike band structure while a polaron lattice gives a metallic structure, but a definite proof of the stability of polarons has not yet been presented [5]. On the other hand, a disordered distribution of bipolarons was shown to produce a metallic band structure with extended states at the Fermi energy [1], thus fulfilling the requirements for a true insulator-to-metal transition.

The presence of extended states in disordered onedimensional chains is a very surprising result that contrasts to Anderson's localization theorem [6]. The precise origin of these states has been explained by $\mathrm{Wu}$ and Phillips [2] based on the random dimer model (RDM). The RDM model was formulated in terms of a tight-binding Hamiltonian, and both diagonal and off-diagonal types of disorder were considered. The existence of unscattered Bloch states by impurities or bond defects in an otherwise perfect monoatomic chain containing $N$ sites was demonstrated, given that the system satisfies the following properties [2]: (i) defects have to occur in pairs (dimers) and have to possess internal symmetry (for example, a pair of identical impurity atoms or a pair of identical distorted bonds around a site), in order to produce resonant scattering for some Bloch states; (ii) there will always exist $\sqrt{N}$ unscattered states, at some energy interval, for an arbitrary concentration of randomly distributed dimers.

Solitons and bipolarons in strictly one-dimensional conjugated polymers usually satisfy those conditions [2]. Phillips and Wu [2] have calculated the reflection coefficients of Bloch states by a single bipolaron in polyaniline and found a resonance to occur at the energy $\sim-0.31 \beta$ (where $\beta \approx 2.5 \mathrm{eV}$ is the characteristic hopping integral of the system), in good agreement with the value obtained by Galvão et al. [1] in their direct density of states calculation of disordered, protonate polyaniline, for the energy where the metallic transition should be observed.

In this work we show that in RDM model internal symmetry is not a necessary condition for dimers to produce near resonant scattering of Bloch states in a onedimensional system. We prove the above statement by calculating reflection coefficients due to scattering by asymmetric dimers and by direct density of states (DOS) calculations of long chains. We apply the present theory to a family of substituted polyanilines. We would like to stress that the derivation shown below is a general procedure for an $A B$-type one-dimensional chain, once it is always possible to renormalize polyaniline-type chains into an $A B$ chain. We have chosen polyanilines in particular for the present application due to the availability of an immense amount of theoretical and experimental work. A very interesting prediction that emerged from our results is the possibility that simple chemical substitution could produce a dramatic increase or decrease in the conductivity by simply inducing the movement of the extended states upward or downward around the Fermi energy, without modification of the $\pi$ network.

The present model is based on the same physical grounds used in the RDM model. We start with a tight-binding monoatomic chain, for which atomic site energies are $\varepsilon_{a}$ and atoms are connected by the hopping $V$. One-electron wave functions are written in terms of 
the atomic basis set as $\psi_{k}=\sum_{n} c_{n}(k)|n\rangle$. The eigenvalue equation $\left(E-\varepsilon_{n}\right) c_{n}=V_{n, n+1} c_{n+1}+V_{n, n-1} c_{n-1}$ gives a dispersion relation $E=\varepsilon_{a}+2 V \cos k$ for the pure monoatomic chain. Following Phillips and $\mathrm{Wu}[2]$, we consider the scattering of a Bloch state by the asymmetric dimer located at sites 0 and 1 , with corresponding site energies $\varepsilon_{b}$ and $\varepsilon_{c}$, and connected by the hopping $\bar{V}$. Expansion coefficients of scattered states can be written as $c_{n}(k)=e^{i k n}+r e^{-i k n}$ for $n \leq 0$ and $c_{n}(k)=t e^{i k n}$ for $n \geq 1$, where $r$ and $t$ are related, respectively, to the reflected and transmitted amplitudes. The reflection coefficient is then easily obtained:

$$
|r|^{2}=1-\frac{4 V^{2} \bar{V}^{2} \sin ^{2} k}{\left[\bar{V}^{2}-V^{2}-W_{b} W_{c}-V\left(W_{b}+W_{c}\right) \cos k\right]^{2}+V^{2}\left[\left(W_{b}-W_{c}\right)^{2}+4 \bar{V}^{2}\right] \sin ^{2} k},
$$

where $W_{b}=\varepsilon_{a}-\varepsilon_{b}$ and $W_{c}=\varepsilon_{a}-\varepsilon_{c}$. Notice that the above expression reduces to the one obtained by Phillips and $\mathrm{Wu}$ [2] when $W_{b}=W_{c}$. This is a general equation for the reflection coefficient due to scattering by an asymmetric dimer, and it will vanish for Bloch states of a system satisfying

$$
\cos k=\frac{W_{b}+W_{c}}{4 W_{b} W_{c}} \frac{\bar{V}^{2}-V^{2}-W_{b} W_{c}}{V} \pm \frac{W_{b}-W_{c}}{4 W_{b} W_{c}} \sqrt{\frac{\left[\bar{V}^{2}-V^{2}-W_{b} W_{c}\right]^{2}}{V^{2}}-4 W_{b} W_{c}} .
$$

This equation is equivalent to that found in the case of a symmetric dimer $\left(W_{b}=W_{c}\right.$ and $\left.\bar{V}=V\right)$, for which resonances satisfy $|\cos k|=\left|W_{b} / 2 V\right| \leq 1$. Within the range of tight-binding parameters that are physically meaningful for conjugated polymers, a small imaginary part of $\cos k$ is found that prevents $|r|^{2}$ from being exactly zero. This is also observed in the symmetric case, since a particular set of parameters may produce $\cos k>1$.

The above results bring the discussion about localization length, that is, how long should a polymer chain be so that near resonant states survive as extended states. A very crude estimate of the possible effect of having a small, finite reflection coefficient would be that the damping of the incident electron density after being scattered by $N$ defects is $T^{N}$ times the incident amplitude, where $T$ is the transmission probability. Taking $T=1-10^{-4}$ (a typical value obtained in our calculations) and admitting $10 \%$ of attenuation of the incident electron amplitude, it would be necessary for the electron to be scattered by $N=(\ln 0.9) / \ln T \approx 1050$ defects, which is much larger than the usual conjugated chain length. Since real materials always have finite chains, in the cases we investigated we could say we found a true resonance. Finally, the existence of $\sqrt{N}$ unscattered states on a chain containing $N$ sites is proved without any assumption on the nature of the defects, thus it should also hold for asymmetric dimers given that a single resonance is found.

Substituted polyanilines have recently been synthesized and characterized [7-11]. Two forms of these polymers are poly(o-methylaniline) (PMA) and poly(2,5dimethylaniline) (PDMA), whose structures are shown in Fig. 1(a), with the appropriate choice of the radicals $R$ and $R^{\prime}$. Both materials present similar behavior of the parent polymer polyaniline: Their reaction with acids leads to the raise of electric conductivity. Methyl-substituted polyanilines are not especially conductive $[7,8]$. The presence of alkyl groups connected to benzene rings adds more disorder in the three-dimensional structure and also enhances steric hindrance that makes the chains less conju- gated, since the rings are displaced from the conjugation plane. Highly conductive materials have been obtained with 2,5-dimethoxy substitution. For our purposes, alkyl substitution represents the simplest test for the theory presented, where the aim is to investigate the influence of substitution on the electronic properties of fully conjugated chains. As the alkyl groups, linked to the carbon rings, do not change the number of $\pi$ electrons nor are they supposed to strongly interact with the $\pi$ network, one could assume that, like in polyaniline, protonation of imine nitrogens induces changes in transport properties.

Bipolarons in PMA are not symmetrical structures, because the six-membered ring is monosubstituted, while in

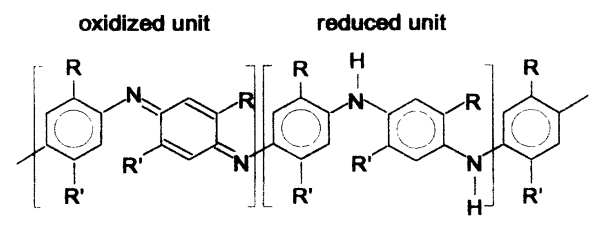

(a)

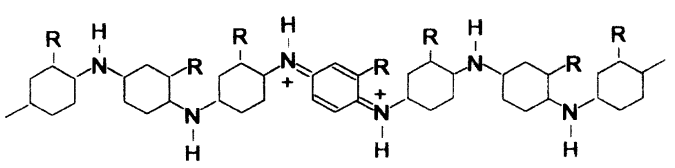

(b)

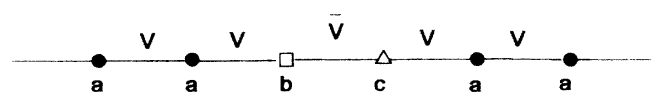

(c)

FIG. 1. (a) Oxidized and reduced units of PAN chains. Emeraldine base, the most conducting form of PAN, is composed of $50 \%$ of each unit. PAN: $R=R^{\prime}=H$; PMA: $R=\mathrm{CH}_{3}, R^{\prime}=H$; PDMA: $R=\mathrm{CH}, R^{\prime}=\mathrm{CH}_{3}$; (b) Fully reduced PMA $\left(R=\mathrm{CH}_{3}\right)$ chain with a bipolaron; and (c) renormalization into a monoatomic chain with a nonsymmetric defect (after Ref. [11]). 
PDMA the symmetry exists. In order to clarify the difference between these polymers, we renormalized both structures into a "monatomic" chain, by the use of a tightbinding Hamiltonian and a two-step decimation process [11]. Note that the carbon to which the ligands are attached is distinguished from the other carbons in the rings by a different site energy [12]. For simplicity, we have considered the presence of a single bipolaron $\left[-\mathrm{NH}^{+}=Q\right.$ $R=\mathrm{NH}^{+}-$] $(Q-R$ represents the substituted quinoid ring) and the polymers in the fully reduced form, as in Fig. 1(b). PMA maps into the monoatomic chain of Fig. 1(c), which presents a nonsymmetric dimer defect.

The calculation of reflection coefficients and DOS evaluations requires choice of tight-binding parameters that best represent the family of polyanilines. These are shown in Table I. Reflection coefficients were calculated for PMA, PDMA, and PAN for comparison purposes, and are depicted in Fig. 2. It is observed that monosubstitution of methyl groups on the benzene rings has a negligible effect in the electronic structure. The transition to metallic regime should occur at the same doping level for both PMA and PAN, as the resonant states are at the energy $\sim-0.3 \beta$. A second ligand attached to carbon rings changes the electronic structure such that two regions of resonant scattering occur in the valence band. The first region of extended states appears in PDMA spectrum around $-0.22 \beta$ and seems to be larger than the ones obtained for PMA and PAN. This feature points to an interesting aspect of the role of substitution in conjugated polymers: ligands added laterally to a conjugated chain can move the energy region where resonance takes place or even suppress resonant scattering without any assumption on their role in keeping or interrupting conjugation. Ligands could be found so that the transition to the metallic state occurs at lower doping than is necessary for the unsubstituted polymer, just by moving the energy at which resonance occurs upward or downward in the spectrum.

As stated above we have also carried out explicit DOS calculations. The DOS of long, finite, ordered, and disordered PAN, PMA, and PDMA chains as a

TABLE I. Hückel parameters for substituted polyanilines [12], in units of $\beta=2.5 \mathrm{eV}$.

\begin{tabular}{lccr}
\hline \hline Carbon site energies & \multicolumn{3}{c}{ Hoppings } \\
\hline $\mathrm{C}-\mathrm{NH}$ & -0.15 & $\mathrm{C}-\mathrm{NH}$ & -0.8 \\
$\mathrm{C}-\mathrm{N}=$ & -0.05 & $\mathrm{C}-\mathrm{N}=$ & -0.8 \\
$\mathrm{C}-\mathrm{CH}_{3}$ & 0.5 & $\mathrm{C}=\mathrm{N}$ & -1.0 \\
Nitrogen site energies & & $\mathrm{C}_{\alpha}-\mathrm{C}_{\beta}{ }^{\mathrm{a}}$ & -0.9 \\
$-\mathrm{NH}-$ & -1.5 & $\mathrm{C}_{\beta}-\mathrm{C}_{\beta}{ }^{\mathrm{a}}$ & -1.1 \\
$-\mathrm{N}=$ & -0.5 & $\mathrm{C}_{\alpha}-\mathrm{C}_{\beta} \mathrm{b}$ & -0.9 \\
$-\mathrm{NH}^{+}=$ & -2.0 & $\mathrm{C}_{\beta}-\mathrm{C}_{\beta}{ }^{\mathrm{b}}$ & -1.0 \\
\hline \hline
\end{tabular}

${ }^{\mathrm{a} Q u i n o i d}$ rings.

bBenzenoid rings.

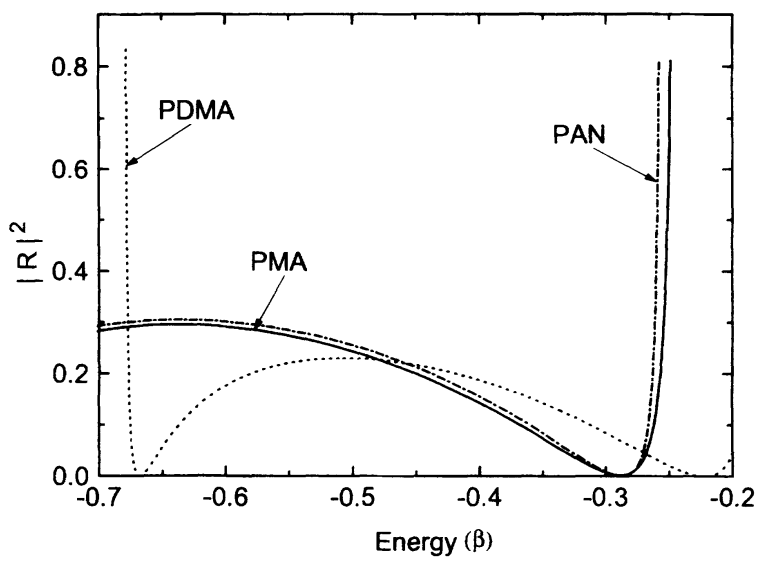

FIG. 2. Reflection coefficients for PAN, PMA, and PDMA as a function of energy (units of $\beta$ ), showing unscattered states at $\sim-0.3 \beta$ (PAN and PMA) and $-0.22 \beta$ (PDMA).

function of bipolaron concentration are obtained using the negative factor counting (NFC) technique [13], coupled to a tight-binding Hamiltonian (Hückel approach). The eigenvectors associated with the Fermi energy region are analyzed through the expansion coefficients $\left\{c_{n}\right\}$ of the molecular orbitals from the usual linear combination of atomic orbitals in the $p_{z}$ basis. The coefficients $\left\{c_{n}\right\}$ are obtained using the inverse iteration method [13]. We have considered chains containing typically 100 rings, which is the average chain length obtained experimentally.

We observed that, similarly to the PAN results reported earlier [1], the ordered PMA and PDMA chains present a gap of $0.4 \beta$. Ordered PMA and PDMA protonated chains (even at $50 \%$ protonation rate) show a small, but finite, gap consistent with a semiconducting behavior. The finite gap is artificially produced by the unrealistic assumption of an ordered polymer. When we consider the case of a disordered distribution of defects, a true metallic behavior appears, as clearly indicated in Figs. 3 and 4. These figures indicate the DOS and the HOMO (highest occupied molecular orbital) wave function expansion coefficients $\left\{c_{n}\right\}^{2}$ for disordered, protonated (50\%) PAN, PMA, and PDMA chains. As we can see the Fermi energy is inside the valence band and the states around the Fermi region are clearly extended, fulfilling the requirements for a $p$-type metal. The energy values for the metallic transition induced by the protonation are in very good agreement with the ones obtained through the reflection coefficients calculations $(-0.35 \beta,-0.35 \beta$, and $-0.26 \beta$, versus $-0.3 \beta,-0.3 \beta$, and $-0.22 \beta$ for PAN, PMA, and PDMA, respectively).

These results strongly support the idea that disorder is the underlying mechanism involved in the metallic transition $[1,2,14]$. The metallic regime is attained only with a disordered distribution of defects. The combined results from the reflection coefficients and DOS calculations for disordered PMA chains prove, beyond doubt, that it is 


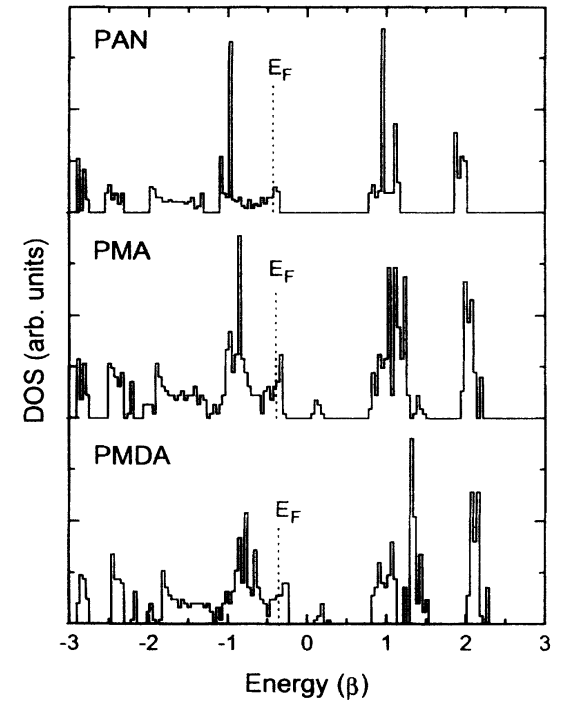

FIG. 3. Density of states (DOS) for a disordered distribution of bipolarons at $50 \%$ protonation. Dotted lines indicate the position of the Fermi energy $\left(E_{F}\right)$.

possible to have near resonant scattering from nonsymmetrical dimers.

It seems that the "anomalous" (in terms of pure monoatomic chains) behavior of conjugated polymers is related to the fact that these systems have bond alternation (an atom connected to the same kind of atoms to its right and left might have different hopping integrals leading to a topological gap). Every conjugated chain can be mapped into a renormalized monoatomic chain. As the renormalized parameters depend on the one-electron energy, they change continuously along the spectrum. This

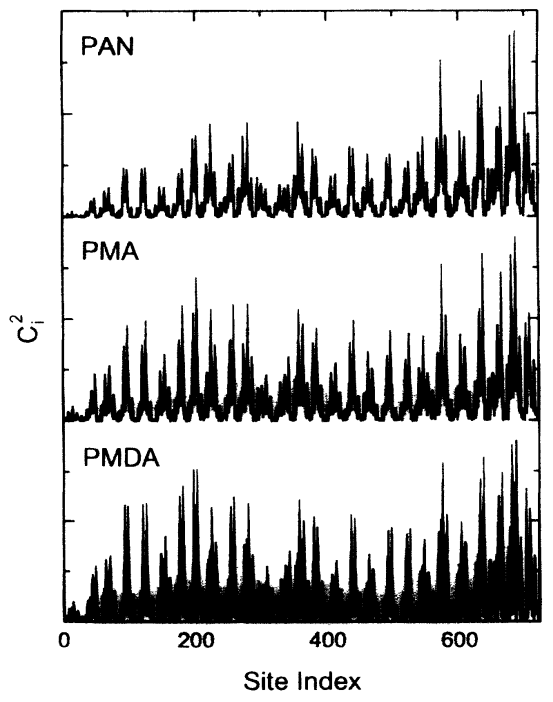

FIG. 4. Squared expansion coefficients $\left\{c_{i}\right\}^{2}$ of highest occupied electronic states at $50 \%$ protonation and a disordered distribution of bipolarons. gives more flexibility to attain the energetic conditions needed for resonant scattering.

In summary, we have demonstrated the existence of extended electronic states at the Fermi energy in protonated, disordered alkyl-substituted polyanilines, which characterizes the insulator-to-metal transition. The lack of symmetry of the charge-trapping defects (bipolarons) does not prevent near resonant scattering to occur, contrary to the model proposed by Phillips and co-workers [2], in which internal symmetry of the dimers is a necessary condition. The results are completely general and hold for every system that can be mapped, by means of renormalization procedures, into a one-dimensional $A B$ chain.

Work supported in part by CNPq, CAPES, FINEP, and FAPESP.

[1] D. S. Galvão, D. A. dos Santos, B. Laks, C.P. de Melo, and M. J. Caldas, Phys. Rev. Lett. 63, 786 (1989).

[2] P. Phillips and H.-L. Wu, Science 252, 1805 (1991); H.-L. Wu and P. Phillips, Phys. Rev. Lett. 66, 1366 (1991); H.-L. Wu, W. Goff, and P. Phillips, Phys. Rev. B 45, 1623 (1992).

[3] S. Stafström, J.-L. Brédas, A. J. Epstein, H. S. Woo, D. B. Tanner, W. S. Huang, and A. G. MacDiarmid, Phys. Rev. Lett. 59, 1464 (1987).

[4] Z. H. Wang, E. M. Scherr, A. G. MacDiarmid, and A.J. Epstein, Phys. Rev. B 45, 4190 (1992); D. Jeon, J. Kim, M.C. Gallagher, and R.F. Willis, Science 256, 1662 (1992).

[5] H. A. Mizes and E. M. Conwell, Phys. Rev. Lett. 70, 1505 (1993); P. Vogl and D. K. Campbell, Phys. Rev. B 41, 12797 (1990).

[6] P. W. Anderson, Phys. Rev. 109, 1492 (1958).

[7] Z. H. Wang, A. Ray, A. G. MacDiarmid, and A. J. Epstein, Phys. Rev. B 43, 4373 (1991).

[8] P. Snauwaert, R. Lazzaroni, J. Riga, and J. J. Verbist, Synth. Met. 18, 335 (1987); E. M. Genies and P. Noel, J. Electroanal. Chem. 296, 473 (1990).

[9] M. C. Gupta and S.S. Umare, Macromolecules 25, 138 (1992).

[10] G. Zotti, N. Comisso, G. D’Aprano, and M. Leclerc, Adv. Mater. 4, 749 (1992).

[11] M.C. dos Santos and J. L. Brédas, Phys. Rev. Lett. 62, 2499 (1989); Synth. Met. 29, E321 (1989), and references therein.

[12] Most data have been taken from A. Streitwieser, Jr., Molecular Orbital Theory (Wiley, New York, 1961), p. 135. Other data have been adapted to account for effects of methyl substitution on benzene rings.

[13] P. Dean, Proc. R. Soc. London Sect. A 254, 507 (1960); J. Ladik, M. Seel, P. Otto, and A. K. Bakhshi, Chem. Phys. 108, 203 (1986).

[14] A. K. Sen and S. Gangopadhyay, Physica (Amsterdam) 186A, 270 (1992); H. Dücker, M. Struck, Th. Koslowski, and W. von Niessen, Phys. Rev. B 46, 13078 (1992); X. Chen and S. Xiong, J. Phys. Condens. Matter 5, 4029 (1993); S. Sil, S. N. Karmakar, R. K. Moitra, and A. Chakrabarti, Phys. Rev. B 48, 4192 (1993). 